[Agr. Biol. Chem., Vol. 27, No. 1, p. 1 7, 1963]

\title{
Studies on Metal Chelate Compounds of Anthraquinone Derivatives
}

\author{
Part I. Magnesium Chelate Compounds of 1-Hydroxy and 1,4- \\ Dihydroxyanthraquinone
}

\author{
By Rikisaku Suemitsu \\ Department of Technical Chemistry, Faculty of Technology, \\ Doshisha University, Kyoto
}

Received May 16, 1962

\begin{abstract}
Structures, absorption spectra, and stabilities of magnesium chelates of 1-hydroxyanthraquinone and 1,4-dihydroxyanthraquinone were examined by the spectrophotometric method. 1-Hydroxyanthraquinone reacts with magnesium in alcoholic solution to form a 2:1 chelate compound (I), while 1,4-dihydroxyanthraquinone forms a 1:1 chelate compound, which is probably a polymer (II) .
\end{abstract}

\section{INTRODUCTION}

Studies on metal chelates have been noticed with keen interests not only in the field of analytical chemistry, but of pharmacy, biology, and high molecular chemistry. For example, 8-hydroxyquinoline ${ }^{1)}$ (oxine), which had originally been used for analytical reagent, its derivatives, and phenazine derivatives $^{2)}$ show antibacterial action. But pyridine derivatives such as 2-methylpyridine-4carboxylic acid ${ }^{3)}$ and 2-butylpyridine-6-carboxylic acid ${ }^{4}$ (fusaric acid) are known to have remarkable injurious action on plantgrowth. Tamari ${ }^{5)}$ reported that injurious effects of these acids on plant growth were caused by their intermolecular chelation with metal ion which are essentially necessary for the formation of Fe-porphyrion oxidase, cytochrome and other enzymes containing metal

1) A. Albert, J. Biochem, 41, 534 (1947).

2) Y. Kawakami, et al., J. Antibiotics, Ser. A, 8, 51 (1955).

3) K. Tamari, Nippon Nogei Kagaku Kaishi, 27, 144 (1953).

4) T. Yabuta, K. Kambe and T. Hayashi, ibid, 10, 1059 (1934).

5) K. Tamari, ibid., 27, 144, 147, 159, 245, 249, 302 (1953). ions.

It is of interest that some of metabolic products produced by microorganisms especially by pathogenic organisms, are found to be chelate reagents such as kojic acid ${ }^{6}$, fusaric $\operatorname{acid}^{7)}$, and hydroxyanthraquinones.

Flumiani $^{8)}$ has reported on the copper chelates of some hydroxyanthraquinones, in which he concluded that the presence of a hydroxyl group at the a-position is decisive for the formation of normal chelate of 2:1, whereas for the formation of an addition compound the presence of a hydroxyl group at the $\beta$-position is decisive.

Recently Korshark ${ }^{9)}$ has reported that coordination chain polymers of quinizarine (1,4dihydroxyanthraquinone) and $\mathrm{Zn}, \mathrm{Cu}, \mathrm{Mn}$, Ni , Co and Cd (bivalent, co-ordination No. 4) were synthesized by treating quinizarin with metal acetylacetonates.

\footnotetext{
6) R.V. Davies, et al., J. Appl. Chem. 9, 368 (1950).

7) K. Tamari, Nippon Nogei Kagaku Kaishi, 26, 223 (1952).

8) Flumiani, Monatsh., 72, 368 (1939); 74, 92 (1942).

9) Korshark, et al., Vysokomolekuyarnye Soedininiya, 2, 492 (1960).
} 
Shibata ${ }^{10}$ found that alcoholic solutions of $a$-hydroxyanthraquinones form beautiful internal complex salts by treating with magnesium acetate.

And this color reaction is commonly used for detecting the position of hydroxyl group in the anthraquinone ring, but it is not clarified in the view of chelate compound.

In this paper, properties of magnesium chelate of 1-hydroxyanthraquinone, which possesses one functional group, was studied spectrophotometrically in ethanol solution, and quinizarin, having two functional groups in it, was also dealt with at the same time.

\section{EXPERIMENTAL}

Apparatus. $\mathrm{pH}$ measurements were carried out with a Yanagimoto glass electrode $\mathrm{pH}$-meter, direct reading type. Measurements of absorbance were carried out with a Shimazu electro-spectrophotometer, Model QB50 , direct reading type.

Materials. (a) Solvents: Since anthraquinone derivatives are insoluble in water, ethanol was used as a solvent. Ethanol was purified by treatment with conc. $\mathrm{H}_{2} \mathrm{SO}_{4}, \mathrm{KOH}$-alkaline $\mathrm{AgNO}_{3}$, and $\mathrm{CaO}$, followed by distillation.

(b) 1-Hydroxyanthraquinone ${ }^{11)}$ was synthesized by means of diazo-reaction of 1 -amino anthraquinone; m.p. $200 \sim 203^{\circ} \mathrm{C}$.

(c) 1,4-Dihydroxyanthraquinone ${ }^{12)}$ (quinizarin) was synthesized by means of condensation of phthalic anhydride with hydroquinone; m.p. $196 \sim 198^{\circ} \mathrm{C}$.

(d) Magnesium Acetate: Special grade for analytical use was used.

Procedure. Preparation of solution for absorption spectral measurements.

(a) $5 \times 10^{-5} \mathrm{M}$ solution of 1-hydroxyanthraquinone was prepared by dissolving $1.12 \mathrm{mg}$ of the reagent in $100 \mathrm{ml}$ of ethanol.

(b) $5 \times 10^{-5} \mathrm{M}$ solution of 1,4-dihydroxyanthraquinone was prepared by dissolving $1.20 \mathrm{mg}$ of reagent in $100 \mathrm{ml}$ of ethanol.

(c) Mg-chelate solution of reagents were prepared as follows. A few drops of saturated ethanol solution

\footnotetext{
10) S. Shibata, J. Am. Chem. Soc., 72, 2789 (1956); J. Pharm. Soc. Japan, 61, 103 (1941).

11) H. Roemer, Ber, 15, 1793 (1882).

12) C. Liebermann, Ann., 212, 10 (1882).
}

of magnesium acctate were added to $5 \mathrm{ml}$ of the reagent.

(d) $\mathrm{pH}$ adjustment: For the absorption spectral measurements, acidty or alkalinity was changed by adding a few drops of ethanol solution of acetic acid or potassium hydroxide, and change in $\mathrm{pH}$ of the solution was measured by a $\mathrm{pH}$-meter.

(e) Molar Ratio Method: To $5 \mathrm{ml}$ of $5 \times 10^{-5} \mathrm{M}$ magnesium acetate ethanol solution, ethanol solutions of the reagent was added at a molar ratio, followed by dilution with ethanol to $10 \mathrm{ml}$. When two, three and four molars weight of reagents are added, the theoretical amounts of reagents which they should combine with $\mathrm{Mg}^{2+}$ were discounted.

\section{RESULTS}

\section{1-Hydroxyanthraquinone-Magensium Chelate.}

(1) Absorption Spectra. In Figs. 1 and 2, absorption curves of both 1-hydroxyanthraquinone and its magnesium chelate compound are shown. The curve represented by $I$ is in acidic (pH 5.6), II in neutral ${ }^{13)}$ (8.3), and III in alkaline ( $\mathrm{pH} 11.9)$ solution. The curves of I and II seem to be nearly the same but III is very different. This phenomenon may be ascribed to changes in the structure caused by ionization. Three kinds of absorption curves of magnesium chelate are shown in Fig. 2, that is, in acidc ( $\mathrm{pH} 4.5)$, neutral (pH 8.1), and alkaline ( $\mathrm{pH} 12.0$ ) solutions. The curves of II and III seem to be nearly the same but $I$ in acidic solution is very different. The band which is charcteristic in chealtes in found in $500 \mathrm{~m} \mu$ both in neutrai and alkaline solution, but not in acidic solution.

(2) Effect of pH Change. The absorption of the magnesium chelate solution in various $\mathrm{pH}$ is shown in Fig. 3. The maximum absorption is shown at $\mathrm{pH}$ of about 8.1 to 8.5 , measured at $500 \mathrm{~m} \mu$.

(3) Composition of the Chelate. The determination of composition of the chelate was carried out by means of continuous variation

\footnotetext{
13) The term "neutral" means an intermediate solution between acidic and alkaline ethanol solutions.
} 


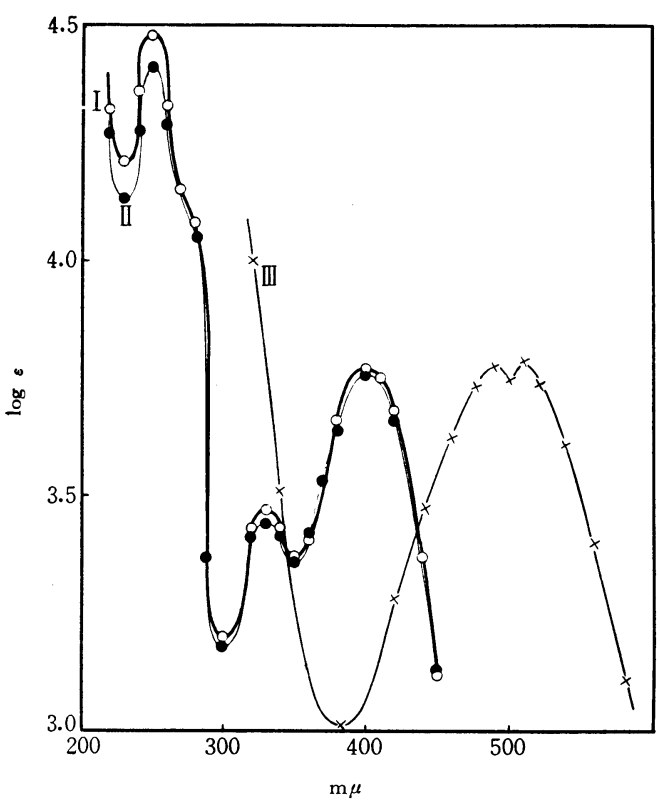

FIG. 1. Absorption Spectra of 1-Hydroxyanthraquinone $\left(5 \times 10^{-5} \mathrm{M}\right)$.

$\begin{array}{lll}\text { Acidic } & \text { I }-\mathrm{O}- \\ \text { Neut. } & \text { III }-0 \\ \text { Alkali. } & \text { II }-\times-\end{array}$

method and molar ratio method.

(a) Continuous Variation Method ${ }^{14)}$ : The molar ratio of the chelate was determined at $500 \mathrm{~m} \mu$ and $\mathrm{pH}$ 8.1. The experimental data clearly indicated that molar ratio of the reagent to magnesium in chelate was 2:1 (Fig. 4).

(b) Molar Ratio Method ${ }^{15)}$ : Under the same condition of ethanol solution at $\mathrm{pH} 8.1$ and $500 \mathrm{~m} \mu$. When the reagent is 2 moles/. $\mathrm{Mg}$, the break was observed; the molar ratio of the reagent to magnesium was found to be 2:1 (Fig. 5) .

II. 1,4-Dihydroxyanthraquinone Magnesium Chelate.

(1) Absorption Spectra. In Figs. 6 and 7, the absorption of both 1,4-dihydroxyanthraquinone and its magnesium chelate are shown. The curves of reagent in acidic and neutral media are almost the same but the one in

14) W.C. Vosburgh, et al., J. Am. Chem. Soc., 63, 437 (1941).

15) A.E. Harvey, et al., ibid., 72, 4488 (1950).

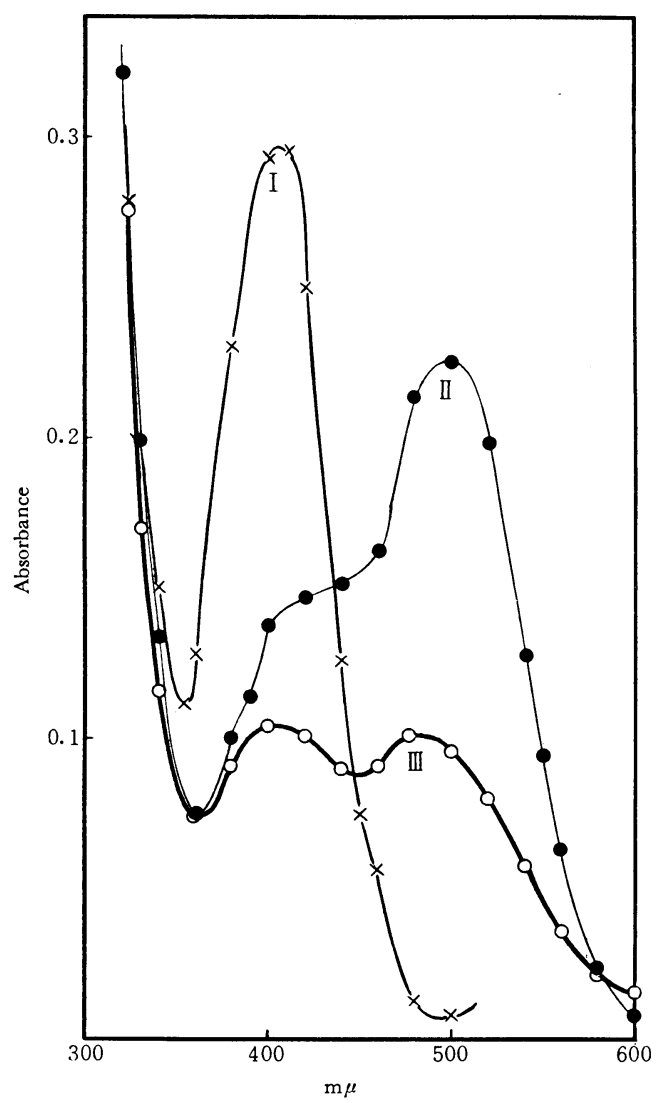

FIG. 2. Absorption Spectra of 1-Hydroxyanthraquinone Magnesium Chelate $\left(5 \times 10^{-5} \mathbf{M}\right)$.

$$
\begin{aligned}
& \text { Acidic } \text { I }->- \\
& \text { Neut. } \text { II }- \\
& \text { Alkali. III }-\mathrm{O}
\end{aligned}
$$

alkaline medium shows a marked difference. On the other hand, the curves of magnesium chelate in acidic medium is very different from what are in neutral and alkaline media. The band which is characteristic in chelation is at $580 \mathrm{~m} \mu$ both in neutral and alkaline solution, but nothing is found in acidic medium.

(2) Effect of $\mathbf{p H}$ Change. The maxmum absorption is shown at a $\mathrm{pH}$ of about 9 as shown in Fig. 8, measured at $580 \mathrm{~m} \mu$.

(3) Composition of the Chelate.

(a) Continuous Variation Method: By this method, the composition of 1,4-dihydroxy- 


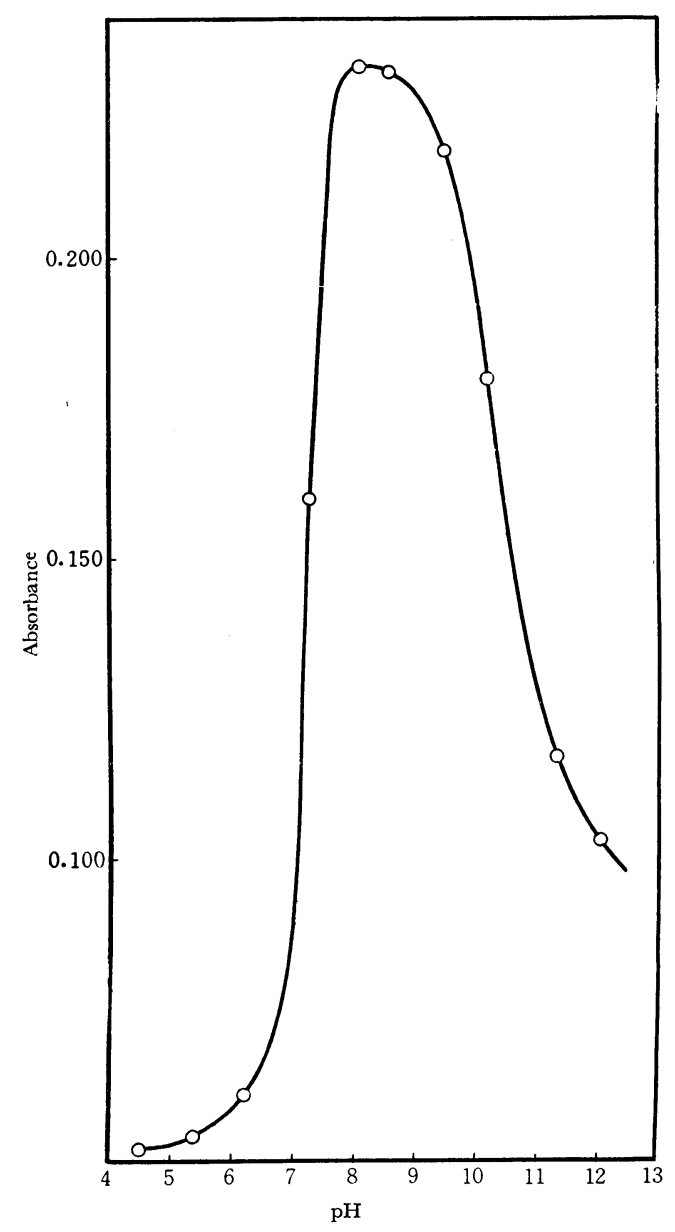

FIG. 3. Effect of $\mathrm{pH}$ Change on 1-Hydroxyanthraquinone Magnesium Chelate $(500 \mathrm{~m} \mu)$.

anthraquinone to magnesium was $1: 1$ at $\mathrm{pH} 9$ and $580 \mathrm{~m} \mu$ (Fig. 9) .

(b) Molar Ratio Method: Under similar conditions, the same results as by the former method (Fig. 10) was obtained.

III. Degree of Dissociation and Dissociation Constansts of the Chelate Compouds. From the molar ratio method, degree of dissociation (a) is calculated from equation (1):

$$
\alpha=\frac{E_{m}-E_{s}}{E_{m}}
$$

16) M. Ishidate and Y. Yamane, J. Pharm. Soc. Japan, 79, 389 (1957).

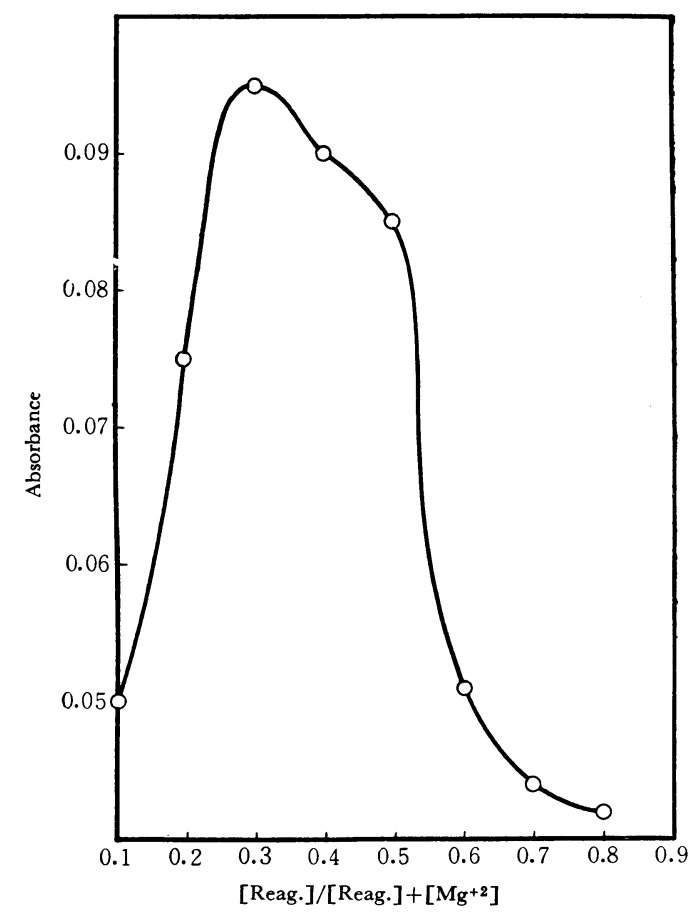

FIG. 4. Absorption Curve of 1-Hydroxyanthraquinone Magnesium Chelate (Job's Method).

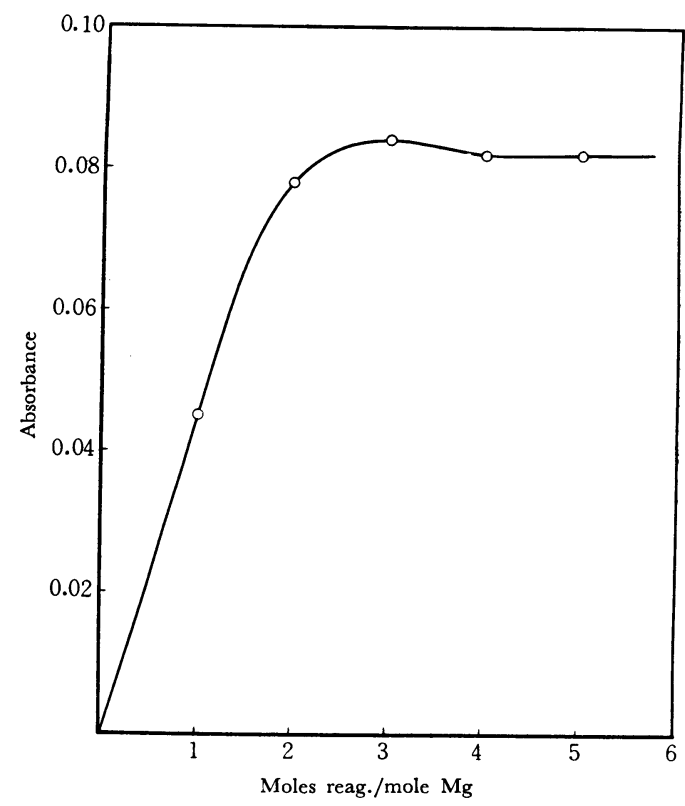

FIG. 5. Absorption Curve of 1-Hydroxyanthraquinone Magnesium Chelate (Molar Ratio Method). 


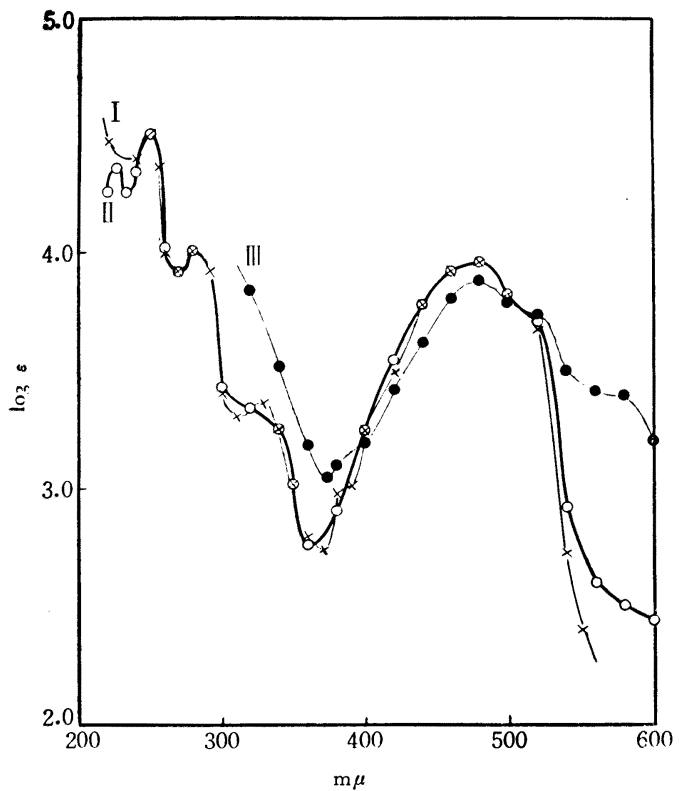

FIG. 6. Absorption Spectra of 1,4-Dihydroxyanthraquinone $\left(5 \times 10^{-5} \mathrm{M}\right)$.

Acidic I $=\times-, \quad$ Neut. II $-0-$

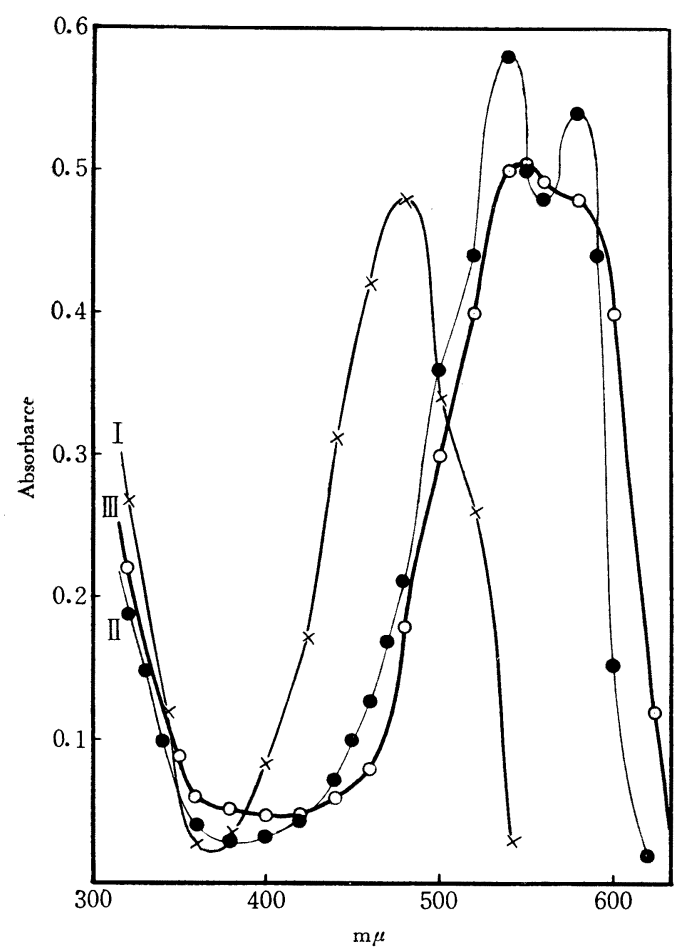

FIG. 7. Absorption Spectra of 1,4-Dihydroxyanthraquinone Magnesium Chelate $\left(5 \times 10^{-5} \mathrm{M}\right)$. Acidic I - $-\infty$, Neut. II -

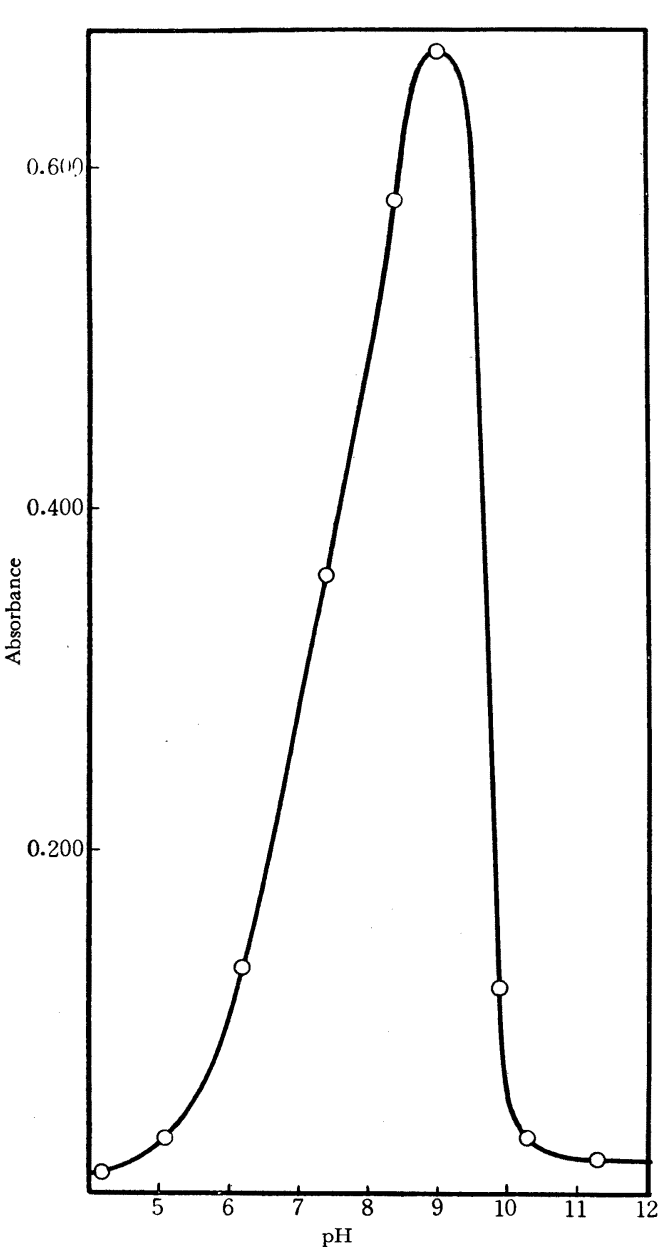

FIG. 8. Effect of $\mathrm{pH}$ Change on 1,4-Dihydroxyanthraquinone Magnesium Chelate $(580 \mathrm{~m} \mu)$. 


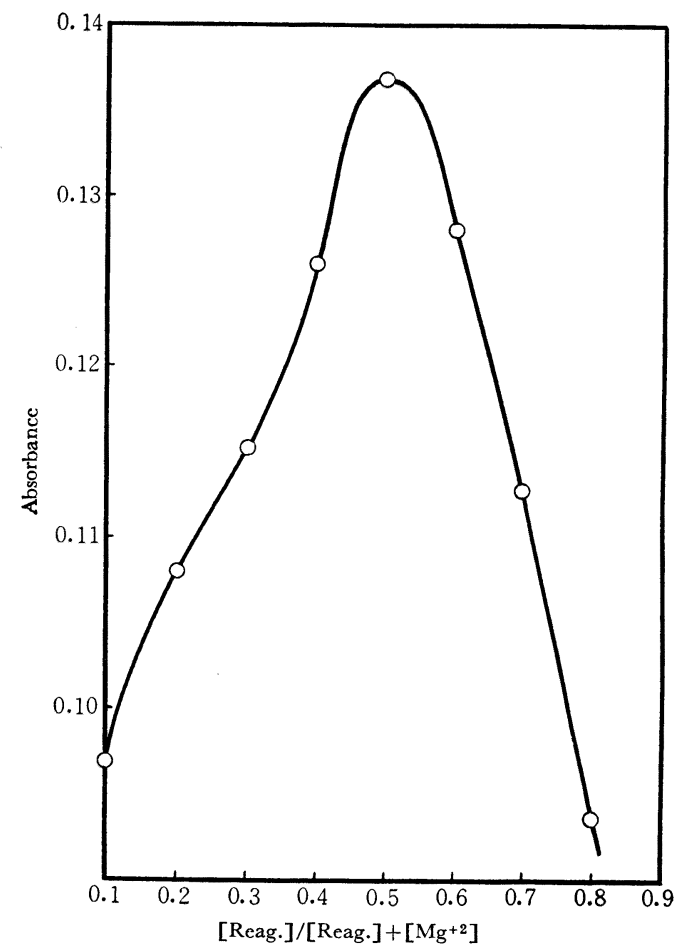

FIG. 9. Absorption Curve of 1,4-Dihydroxyanthraquinone Magnesium Chelate (Job's Method).

where $E_{m}$ is the maximum extinction and $E_{s}$ is the extinction at the stoichiometric molar ratio of the reagent to magnesium in the chelate.

Since the ratio of the reagent to magnesium is $2: 1$, the equilibrium constant may be written as

$$
K=\frac{\alpha(2 \alpha c)^{2}}{1-\alpha} \quad c: \text { total concentration }
$$

Similarly, in the case of $1: 1$ chelate, $K$ is obtained as follows.

$$
K=\frac{\alpha^{2} c}{1-\alpha}
$$

The degree of dissociation (a) and dissociation constant $(K)$ of magnesium chelate of 1-hydroxyanthraquinone and 1,4-dihydroxyanthraquinone calculated from the formulas (1), (2), and (3) are shown in Table I.

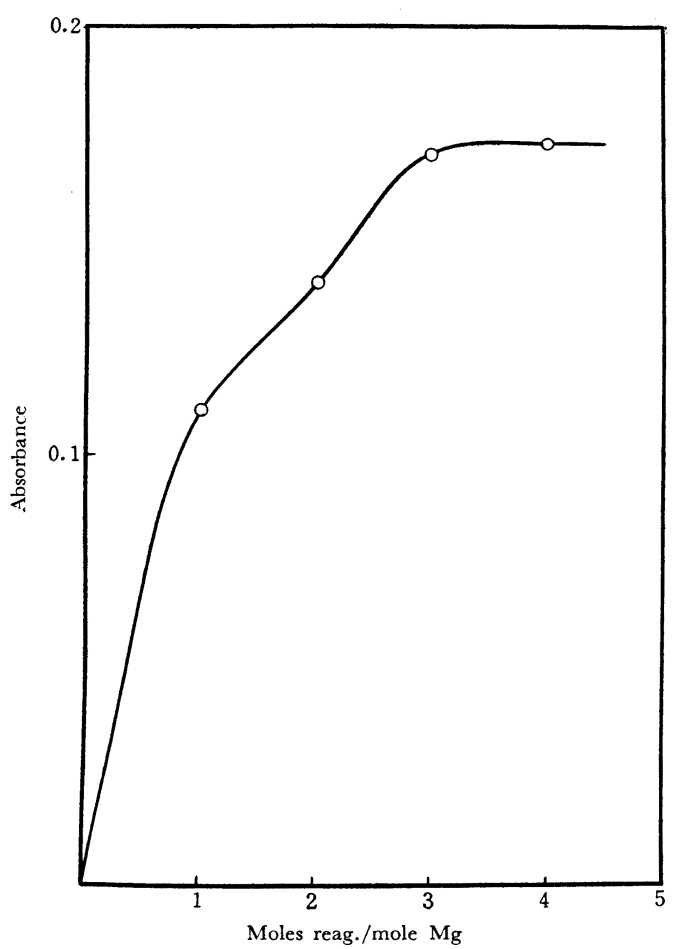

FIG. 10. Absorption Curve of 1-Hydroxyanthraquinone Magnesium Chelate (Molar Ratio Method).

TABLE I.

$\begin{array}{lcccc}\text { Compound } & \boldsymbol{\alpha} & \text { Reag. : Mg } & -\log K & \mathrm{pH} \\ \begin{array}{l}\text { 1-Hydroxy- } \\ \text { anthraquinone }\end{array} & 0.06 & 2: 1 & 11.64 & 8.1 \\ \begin{array}{l}\text { 1,4-Dihydroxy- } \\ \text { anthraquinone }\end{array} & 0.38 & 1: 1 & 4.93 & 9.0\end{array}$

IV. Structure of the Chelate. From the results obtained here, 1-hydroxyanthraquinone forms a stable six membered, 2:1, normal chelate compound, but 1,4-dihydroxyanthraquinone forms a 1:1 chelate compounds; and structures of [I] and [II] are assigned.

\section{DISCUSSION AND CONCLUSION}

It is well known that hydroxyanthraquinones are produced by microorganisms and some of them are produced by pathogenic organisms as metabolic products. The question in regards to the role played by the 

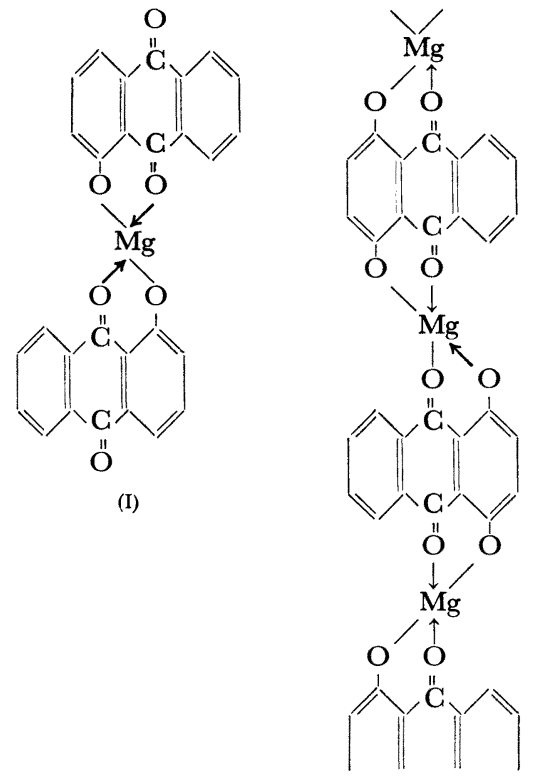

(II)

pigments in vivo has not been clarified yet. a-Hydroxyanthraquinone possesses one functional group, a hydroxyl peri to carbonyl, forming a chelate ring. Raistrick ${ }^{17)}$ has reported a general rule concerning the position of substituents in the anthraquinone ring, in which pigments produced by micro-organisms have at least one $a$-hydroxyl group in it and many pigments have hydroxyl groups both at 4 and 5 position. In this paper, magnesium chelates of 1-hydroxyanthraquinone having one functional group and 1,4-dihydroxyanthraquinone having two functional groups were studied by spectrophotometric method concerning their properties and compositions in solution, as well as their dissociation constants. In neutral and alkaline media 1-hydroxyanthraquinone forms a six-membered chelate ring, indicating the molar ratio of 2:1; 1,4-dihydroxyanthraquinone forms a chelate believed to be a polymer with molar ratio of $1: 1$ in neutral and alkaline media. 1-Hydroxyanthraquinone forms a fairly stable magnesium chelate, showing the dissociation constant of 11.64 .

In these results, it might be said that there are some relationship between the formation of chelate and pathogeny in the plant tissue.

Acknowledgement. The author is indebted to Mr. K. Shiba for his help throughout the course of this work. 